\title{
IMAGE DE-NOISING AND RESTORATION USING WAVELET TRANSFORM
}

\author{
A. Procházka* ${ }^{*}$ I. Šindelářová ${ }^{\dagger}$, and J. Ptáček* \\ * Prague Institute of Chemical Technology, Department of Computing and Control Engineering \\ Technická 1905, 16628 Prague 6, Czech Republic \\ Phone: (+420) $224354198 *$ Fax: (+420) $224355053 *$ E-mail: \{A.Prochazka, J.Ptacek\}@ @ieee.org \\ $\dagger$ University of Economics, Department of Econometrics \\ W. Churchill Sq. 4, 13067 Prague 3, Czech Republic \\ Phone: (+420) $224095443 *$ Fax: (+420) $224095423 *$ E-mail: ISin@vse.cz
}

Keywords: Wavelet transform, image de-noising, restoration, interpolation, biomedical image processing

\begin{abstract}
Image de-noising and restoration represent basic problems in image processing with many different applications including engineering, reconstruction of missing data during their transmission and enhancement of biomedical structures as well. This problem occurs also in filling-in blocks of missing or corrupted data. The paper presents the use of Wavelet transform in this area including its application for image decomposition and rejection of its components at first. The main part of the paper is then devoted to methods of restoration of missing image blocks by the search of similar structures of a given image in the Wavelet domain space and comparison of this approach with iterated Wavelet interpolation and predictive image modelling. Proposed methods are verified for simulated images and then applied for processing of magnetic resonance images.
\end{abstract}

\section{Introduction}

Wavelet transform (WT) represents a general tool for decomposition and reconstruction of multi-dimensional signals for their analysis, resolution enhancement and further processing $[5,8,10,11]$. The first part of the paper is devoted to the summary of basic properties of Wavelet functions both in analytical and recurrent forms for signal decomposition and perfect reconstruction.

The subsequent part of the paper presents algorithms for signal and image decomposition enabling the following signal denoising into selected levels using either global or local threshold limits [11].

The main part of the paper is devoted to the use of Wavelet transform for restoration of missing image blocks $[4,9,14]$ by the search of similar structures using block features in the timescale space. Results if this method are then compared with that achieved by iterated Wavelet interpolation method [3] and autoregressive forward and backward prediction by image rows and columns $[1,2,6]$.

\section{Wavelet transform in signal processing}

Signal Wavelet decomposition using Wavelet transform provides an alternative to the short-time Fourier transform (STFT) for signal analysis $[5,11]$ resulting in signal decomposition into two-dimensional functions of time and scale. The main benefit of the WT over the STFT is in its multi-resolution time-scale analysis ability.

Wavelet functions used for signal analysis are derived from the initial function $W(t)$ forming basis for the set of functions

$W_{m, k}(t)=\frac{1}{\sqrt{a}} W\left(\frac{1}{a}(t-b)\right)=\frac{1}{\sqrt{2^{m}}} W\left(2^{-m} t-k\right)$

for discrete parameters of dilation $a=2^{m}$ and translation $b=k 2^{m}$. Wavelet dilation closely related to its spectrum compression enables local and global signal analysis. An example of an analytically defined Wavelet function is presented in Fig. 1.
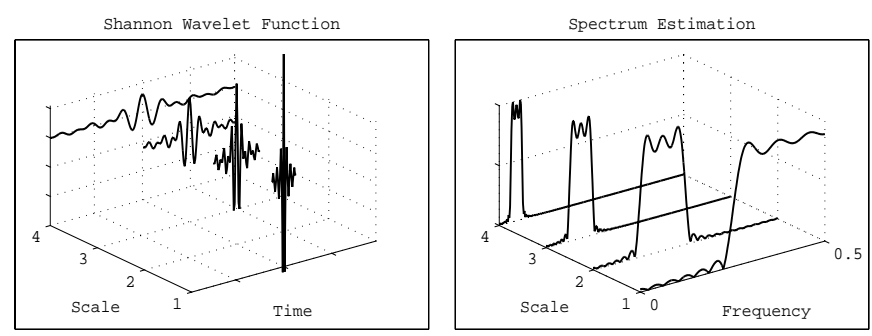

Figure 1: Fundamental Shanon Wavelet function derived from the initial (mother) function defined by the analytical relation $W(t)=\sin (\pi t / 2) \cos (3 \pi t / 2) /(\pi t / 2)$ and the effect of its dilation to its spectrum compression

Wavelet functions can be defined either in the analytical form or by solution of dilation equations. In the case of Daubechies Wavelet functions [5] with four coefficients, the initial Wavelet function is defined by solution of equation

$$
\begin{aligned}
W(x)= & -c_{3} \phi(2 x)+c_{2} \phi(2 x-1) \\
& -c_{1} \phi(2 x-2)+c_{0} \phi(2 x-3)
\end{aligned}
$$

where $\phi(x)$ is the Scaling function. Coefficients of this relation are chosen carefully in order to generate Wavelets with special properties. The basic dilation equation has the form

$$
\begin{aligned}
\phi(x)= & +c_{0} \phi(2 x)+c_{1} \phi(2 x-1) \\
& +c_{2} \phi(2 x-2)+c_{3} \phi(2 x-3)
\end{aligned}
$$


The solution of this dilation equation can be obtained by the iterative algorithm evaluating

$$
\begin{aligned}
\phi_{j}(x)= & +c_{0} \phi_{j-1}(2 x)+c_{1} \phi_{j-1}(2 x-1) \\
& +c_{2} \phi_{j-1}(2 x-2)+c_{3} \phi_{j-1}(2 x-3)
\end{aligned}
$$

for $j=1,2, \cdots$ (see Fig. 2) until $\phi_{j}(x)$ becomes close to $\phi_{j-1}(x)$ in a selected norm. It is possible to start from a box
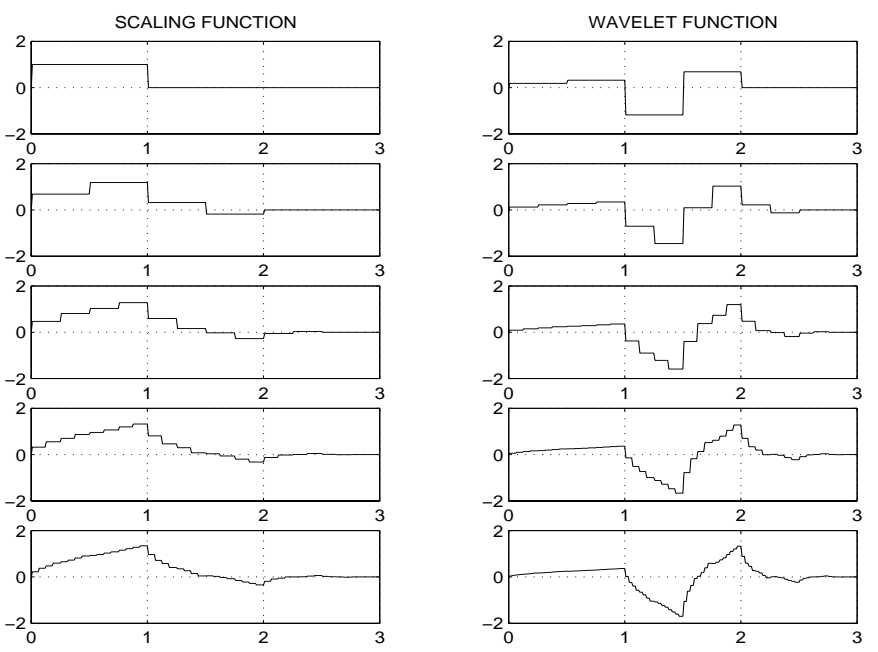

Figure 2: Recurrent solution of dilation equations

function $\phi_{0}(x)=1$ for $x \in(0,1)$ and $\phi_{0}(x)=0$ elsewhere. Scaling and Wavelet functions must satisfy several conditions to enable perfect signal reconstruction - conservation of area condition, accuracy and the orthogonality condition [5].

Coefficients of Wavelet transform can be evaluated using Mallat decomposition tree presented in Fig. 3 for the twodimensional case and the first level of decomposition. At first each column of the image matrix is convolved with the highpass half-band filter (Wavelet function) and the complementary low-pass half-band filter (Scaling function) followed by downsampling. Then the same process is applied to all rows of the image matrix. Each step of the image decomposition results in four image matrices with the number of rows and columns reduced to the half of that of the original matrix.

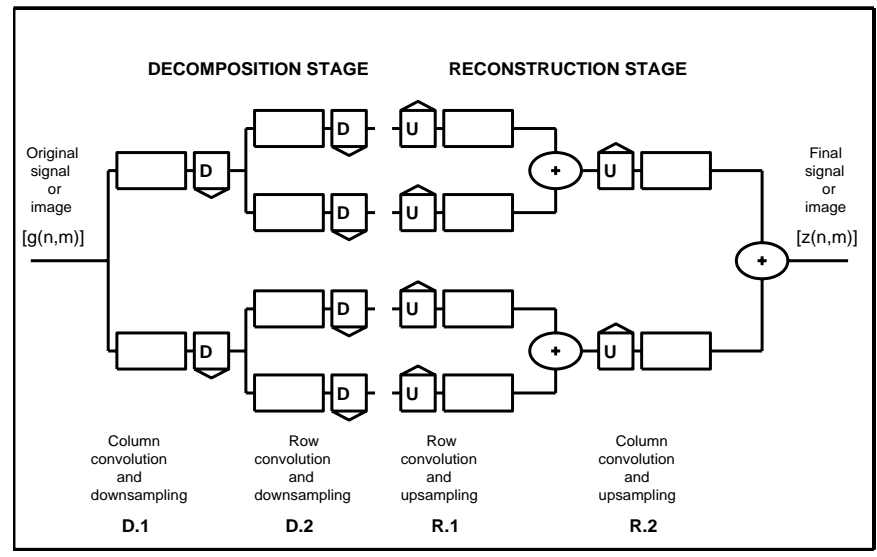

Figure 3: Image Wavelet decomposition tree using column and row two-dimensional signal decomposition with downsampling in each stage followed by image reconstruction
The result of one step image decomposition for a simulated image is presented in Fig. 4. One step of the discrete Wavelet transform decomposition of an image $\mathbf{g}(n, m)$ provides coefficients $\mathbf{c}(k)$ that can be ordered in one row vector containing column representation of 4 subimage in each decomposition level.

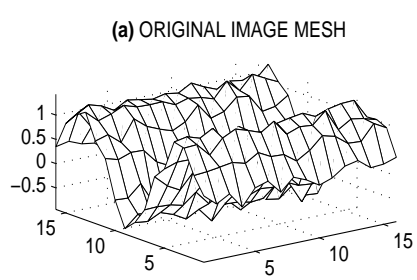

(c) APPROXIMATION IMAGE PART MESH

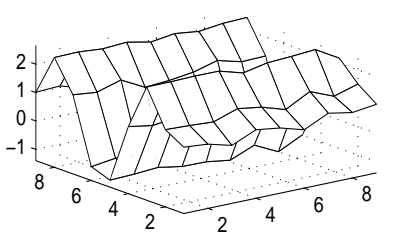

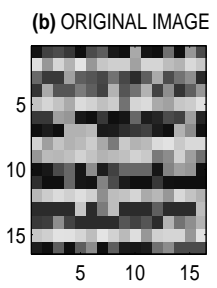

(d) DWT IMAGE DECOMPOSITION

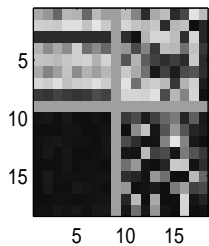

(e) DWT IMAGE COEFFICIENTS

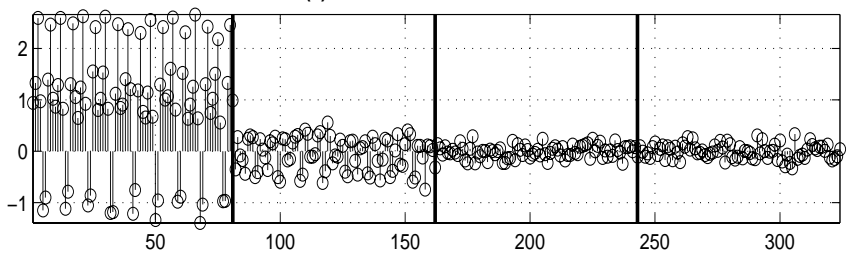

Figure 4: Process of simulated image decomposition presenting (a) original image, (b) its pixel representation, (c) the low pass image component after the image Wavelet decomposition, (d) the first level of image decomposition, and (e) Wavelet coefficients of the original image divided into groups corresponding with image decomposition components

Coefficients $\mathbf{c}(k)$ evaluated in image decomposition stage can be used for image analysis, feature extraction, image denoising, restoration or compression.

The basic metod used for Wavelet signal processing is often based upon global or local Wavelet coefficient thresholding, filtering or modification before the following signal reconstruction. The thresholding process assume estimation of appropriate threshold limits [12] and the resulting algorithm includes

- Signal decomposition using a selected Wavelet function up to the given level and evaluation of Wavelet transform coefficients $\{c(k)\}_{k=0}^{N-1}$

- The choice of threshold limits $\delta$ for each decomposition level and modification of its coefficients for $k=$ $0,1, \cdots, N-1$ using for instance the soft thresholding for evaluation of values

$$
\bar{c}_{s}(k)= \begin{cases}\operatorname{sign} c(k)(|c(k)|-\delta) & \text { if }|c(k)|>\delta \\ 0 & \text { if }|c(k)| \leq \delta\end{cases}
$$

- Signal reconstruction from modified Wavelet transform coefficients

Results of this process depend on the proper choice of Wavelet functions and the selection of threshold limits. 


\section{Image restoration}

There are many possibilities of filling-in missing or corrupted image blocks. The goal of this study is in the analysis of two methods based upon Wavelet transform to perform this task and comparison of results with a selected linear predictive method.

(i) The algorithm of image Subregions Feature Extraction and Classification (SFEC) stands for the first method. It operates with the corrupted block of a two-dimensional signal and searches for a similar block within the whole image to replace corrupted parts of the image by its subregion having the closest boundary components. This search has been applied to the magnetic resonance (MR) image after its Wavelet decomposition (see Fig. 5) to find the most significant missing block boundary features. The pixels around the defect are used as an evidence of the probable structure of unknown pixels and are used for extraction and classification of image subregions.

Resulting algorithm consists of the following steps:

- Extraction of corrupted image boundary features

- The search of the similar structure using the sliding window of the same size as as the corrupted image block

- Selection of the block with the closest features and replacement of the corrupted block
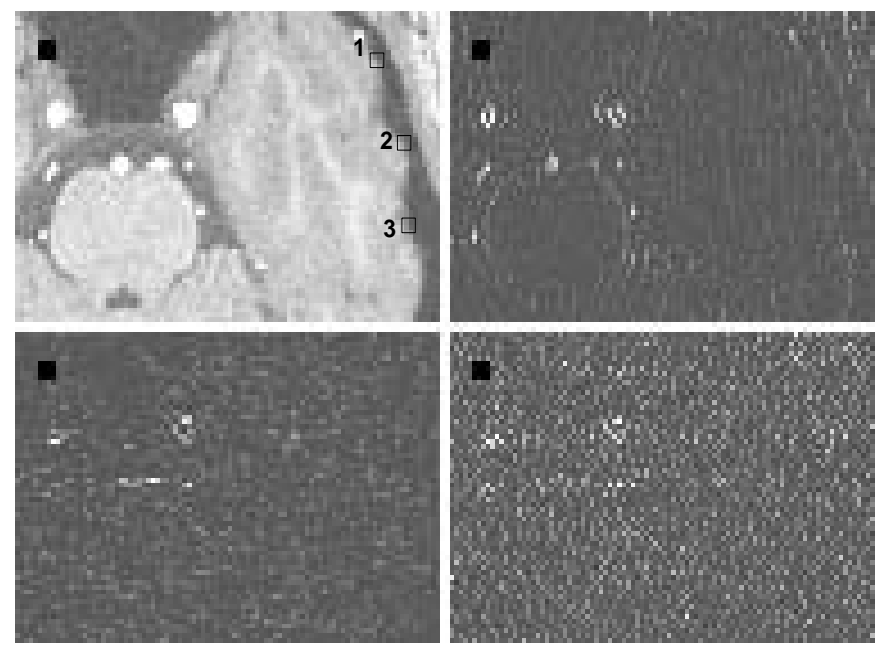

Figure 5: The first stage of the MR image decomposition by Wavelet transform according to Fig. 3 of size $256 \times 191$ pixels with the corrupted part (black) and its closest regions

To find similar structures a sliding block of the same size as the defect region is taken and moved through the image. At each position, except for ones where the sliding block overlaps the defect, the two rows and columns of pixels around the border of the sliding block are placed in a vector $\mathbf{s}$. The reference vector d contains two rows and columns of pixels around the border of the defect block. Then it is possible to calculate the mean square error between the two border vectors

$$
\mathbf{S} \mathbf{1}=\frac{1}{N}(\mathbf{d}-\mathbf{s})^{T}(\mathbf{d}-\mathbf{s})
$$

where $N$ is the number of pixels in each vector. The block or blocks of an image which have the lowest border-pixels mean square error $\mathbf{S 1}$ are the most similar blocks of the two dimensional signal signal.

An alternative for the similarity estimation represented by the mean square error between two matrices of pixel values of selected parts of an image is defined by relation

$$
\mathbf{S 2}=\frac{1}{M N} \sum_{i=1}^{M} \sum_{j=1}^{N}\left(\mathbf{I}_{\mathbf{D}}(i, j)-\mathbf{I}_{\mathbf{S}}(i, j)\right)^{2}
$$

where $\mathbf{I}_{\mathbf{D}}$ and $\mathbf{I}_{\mathbf{S}}$ are the $M \times N$ matrices representing selected image parts.

\begin{tabular}{|l||c|c|c|}
\hline$\left(\times 10^{-3}\right)$ & 1st block & 2nd block & 3rd block \\
\hline \hline S1 & 0.7168 & 0.7389 & 0.7474 \\
\hline S2 & 1.8136 & 1.9377 & 1.9461 \\
\hline
\end{tabular}

Table 1: Values of $\mathbf{S 1}$ and $\mathbf{S 2}$ for three most similar blocks of the MR image to its corrupted part

Table 1 shows the $\mathbf{S 1}$ values for the three most similar blocks to the selected one of the chosen magnetic resonance (MR) image. The first block has the lowest value of $\mathbf{S 1}$ and therefore it is taken as the most similar. The second one represents the next best fit etc. The calculation of the $\mathbf{S 2}$ values, which are also shown in Table 1, indicate that the SFEC method is justified since the values of $\mathbf{S 1}$ and $\mathbf{S 2}$ show similar trends.

Using the algorithm described above the corrupted part of the MR image can be restored. The most similar part to the corrupted one is found using the $\mathbf{S 1}$ values after the Wavelet transform decomposition of the original image. The best results are obtained by searching for similar parts in the first level of Wavelet decomposition. The result is presented in Fig. 5. This level contains the most energy of the original signal.

The components of the decomposed image are then reconstructed. The defect region is replaced by the block that has the lowest value of S1. The result of the restoration of the corrupted part is presented in Fig. 6. The surrounding of the corrupted block is the closest to the surrounding of the most similar one.

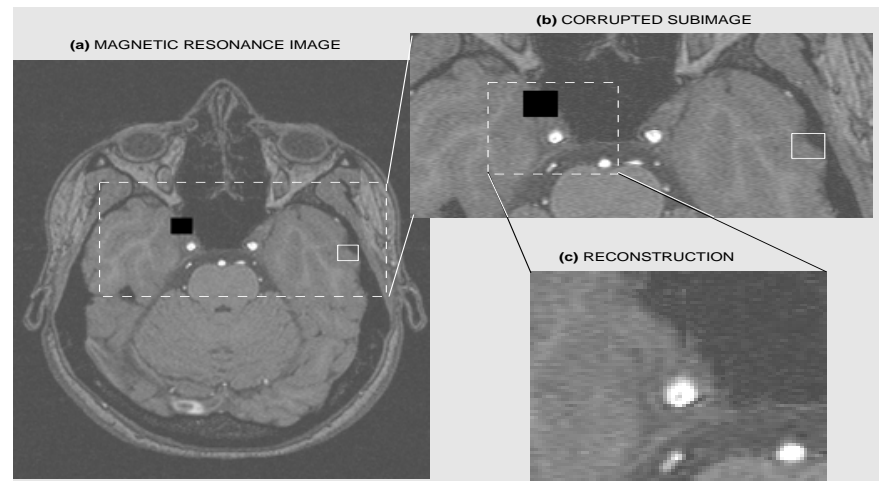

Figure 6: Magnetic resonance image reconstruction presenting (a) the original image with its corrupted part and the suggested similar region using Wavelet decomposition, (b) detail part of regions of interest, and (c) reconstructed image component 

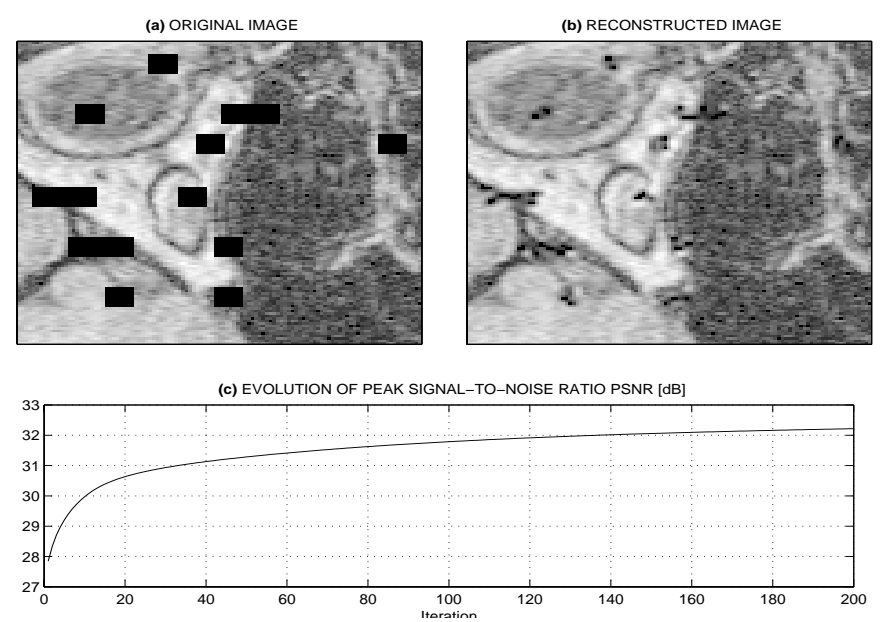

Figure 7: Iterated wavelet interpolation

(ii) The Iterated Wavelet Interpolation Method (IWIM) stands for the second method that can be used for interpolation inside corrupted image regions as presented in Fig. 7. This method assumes localization of corrupted image regions at first and it consists of the following iteration steps:

- Image Wavelet decomposition into a selected level and thresholding of resulting coefficients

- Image reconstruction and transformation of the resulting image to preserve image values outside its corrupted regions creating the new image for the further iteration step

(iii) The Predictive Image Modelling (PIM) described in [2, 6] forms another method for image blocks reconstruction using an autoregressive method. Fig. 8 presents a part of real magnetic resonance image of the human brain, the same signal with an artifact and signal after its reconstruction using the following algorithmic steps:

- Estimation of autoregressive parameters for signal prediction in each image row using image values to the left and to the right of the corrupted block boundary

- Estimation of autoregressive parameters for signal prediction in each image column using image values to the top and to the bottom of the corrupted block boundary

- Prediction of each pixel inside the corrupted image block using for models described in the previous steps and averaging of resulting values (a)

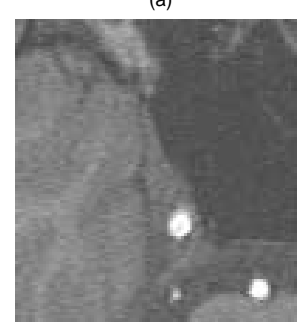

(b)

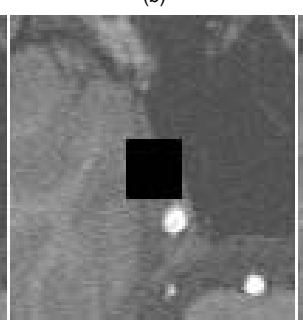

(c)

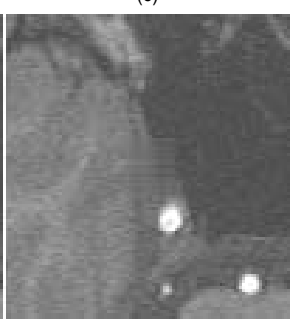

Figure 8: Results of real image restoration using predictive image modelling including (a) original image, (b) image with its corrupted part, and (c) image after reconstruction

\section{Results}

The paper describes selected ways of the Wavelet transform application to image blocks reconstruction and their comparison with a simple autoregressive method.

Fig. 9 presents selected results of a real magnetic resonance image processing by various methods described above using the same corrupted image area including a sharp edge in this case. The goal of such a comparison is in the study of possibilities to restore image artifacts [7]. (o) Corrupted Image

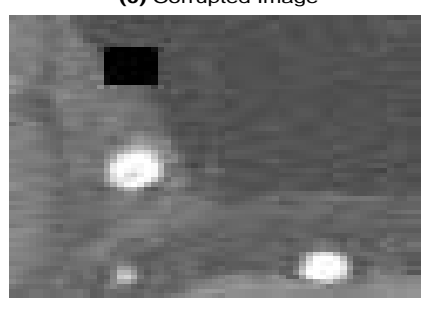

(2) IWIM

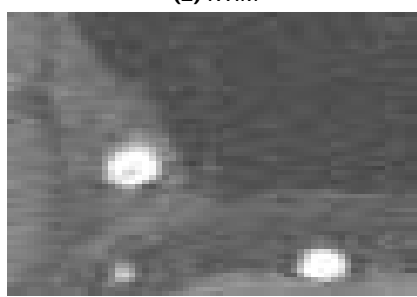

(1) SFEC

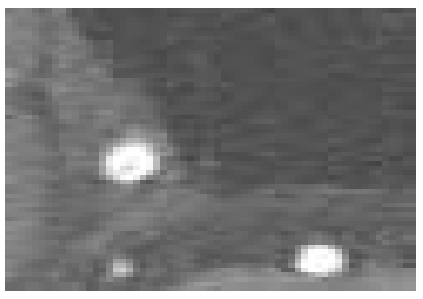

(3) PIM

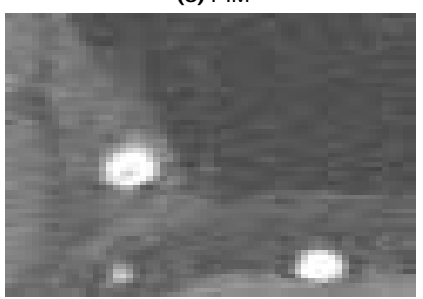

Figure 9: Corrupted image and results of its restoration by (1) SFEC - Subregions Feature Extraction and Classification method, (2) IWIM - Iterated Wavelet Interpolation Method, and (3) PIM - Predictive Image Modelling

Numerical comparison of the same image region processing is presented in Table 2. Results in Table 2 present the PSNR (peak signal-to-noise ratio) between two images (in decibels) and the sum of squared errors $(S S E)$ for methods described above. $P S N R$ is commonly used to measure the difference between two images using the $M S E$ as the root mean square difference between two images for evaluation of

$$
P S N R=20 * \log 10(M / M S E)
$$

where $M$ is the largest possible value of the signal.

Each method has its advantages for restoration of specific image artifacts and replacement of image missing parts and Table 2 should be looked upon from this point of view only [7, 14]. Each two dimensional signal requires a different method for its processing according to its features.

\begin{tabular}{|c|c|c|c|c|c|}
\hline & \multicolumn{2}{|c|}{ Corrupted Image } & \multicolumn{2}{|c|}{ Recovered Image } \\
\hline & & $P S N R[d B]$ & SSE & $P S N R[d B]$ & SSE \\
\hline$\overline{1}$ & SFEC & \multirow{3}{*}{44.28} & \multirow{3}{*}{8.5528} & 52.50 & 0.0552 \\
\hline 2 & IWIM & & & 53.30 & 0.0526 \\
\hline 3 & PIM & & & 52.83 & 0.0532 \\
\hline
\end{tabular}

Table 2: Comparison of image restoration for magnetic resonance subimage processing corresponding to results and methods presented in Fig 9 
Methods presented above were moreover compared with further simple methods presented in [6, 7] including

1. Bilinear Interpolation - BLI (linear interpolation both in the horizontal and in the vertical direction)

2. Triangular Surface Interpolation - TSI (interpolation based on Delaunay triangulation)

3. Matrix Moving Average - MMA (estimation of image pixels as arithmetic means of neighbouring pixels)

These methods were verified for simulated two-dimensional harmonic signals with additive random components at first. All methods were then applied for processing of selected real two dimensional signals representing magnetic resonance images with different artifacts.

Simple methods based upon a limited region of interest are very fast and easy to implement but they provide good results for restoration of low-frequency component only. In the case we need higher frequency image restoration it is useful to apply more complicated linear, nonlinear or statistical models both in the original image domain or after the application of the appropriate transform functions.

In general it is possible to summarize that methods of image restoration based upon the Wavelet transform are very powerful with many possibilities of their modifications taking into account specific features of regions of interests and various methods of their comparison.

\section{Conclusion}

The paper presents selected algorithms allowing image components reconstruction using Wavelet transform features. In the first case boundary features obtained after image Wavelet transform are used. This approach takes into account the low-pass image frequency components providing more reliable results comparing to that using the original image. In the second case a modified Wavelet denoising algorithm is used for restoration of image components. General algorithms described in the paper were applied to magnetic resonance images processing.

Wavelet transform becomes a potential very efficient mathematical tool in digital image processing. Although the utilization of Wavelet transform is not very easy, methods based on this tool give very useful and interesting results in many engineering areas.

Further studies will be devoted to more detail analysis of this approach and its comparison with other nonlinear methods [13, 7, 9, 3] of image restoration. These methods include also Bayesian methods which represent useful tool for highfrequency image components restoration.

\section{Acknowledgments}

The work has been supported by the Research Grant of the Faculty of Chemical Engineering CEZ: MSM 223400007. All real MR images were kindly provided by the Faculty Hospital Královské Vinohrady in Prague.

\section{References}

[1] S. Armstrong, A.C. Kokaram, and P. Rayner. Reconstructing missing regions in colour images using multichannel median models. In IXth European Signal Processing Conference EUSIPCO-98, pages 1029-1032. European Association for Signal Processing, 1998.

[2] W. Etter. Restoration of Discrete-Time Signal Segments by Interpolation Based on the Left-Sided and Right-Sided Autoregressive Parameters. IEEE Transaction on Signal Processing, 44(5):1124-1135, May 1996.

[3] Onur G. Guleryuz. Iterated Denoising for Image Recovery. In Data Compression Conference (DCC '02), Snao Bird, Utah. IEEE, 2002.

[4] A. D. Kulkarni. Computer Vision and Fuzzy-Neural Systems. Prentice Hall PTR, Upper Saddle River, 2001.

[5] D. E. Newland. An Introduction to Random Vibrations, Spectral and Wavelet Analysis. Longman Scientific \& Technical, Essex, U.K., third edition, 1994.

[6] J. Ptáček and A. Procházka. Autoregressive Modelling in Rejection of Image Artefacts. In 13th International Conference on Process Control, Štrbské Pleso. The Slovak Society of Cybernetics and Informatics, 2001.

[7] J. Ptáček and A. Procházka. Restoration of Image Artefacts. In International Conference on Information Engineering and Process Control, Prague, CZ. Czech Technical University in Prague, 2001.

[8] J. Ptáček, I. Šindelářová, A. Procházka, and J. Smith. Wavelet Transform in Signal and Image Resolution Enhancement. In 16th International Conference on Scientific Computing ALGORITMY 02, Podbanske, SK. The Slovak Technical University, 2002.

[9] S. D. Rane, G. Sapiro, and M. Bertalmio. Structure and Texture Filling-In of Missing Image Blocks in Wireless Transmission and Compression Applications. IEEE Trans. on Image Processing, 12(3):296-303, 2003.

[10] O. Rioul and M. Vetterli. Wavelets and Signal Processing. IEEE SP Magazine, pages 14-38, October 1991.

[11] G. Strang. Wavelets and Dilation Equations: A brief introduction. SIAM Review, 31(4):614-627, Dec. 1989.

[12] G. Strang and T. Nguyen. Wavelets and Filter Banks. Wellesley-Cambridge Press, 1996.

[13] A. B. Suksmono and A. Hirose. Interferometric SAR Image Restoration Using Monte Carlo Metropolis Method. IEEE Transactions on Signal Processing, 50(2):290-298, February 2002.

[14] Z. Wang, Y. Yu, and D. Zhang. Best Neighborhood Matching: An Information Loss Restoration Technique for Block-Based Image Coding Systems. IEEE Transactions on Image Processing, 7(7):1056-1061, July 1998. 